\title{
The ups and downs of cocaine in the NAc
}

The nucleus accumbens (NAc) is known to be important for the rewarding effects of cocaine, but little is known about how the subtypes of projection neurons in this region contribute to the effect. Now, Nestler and colleagues show that signalling through the brain-derived

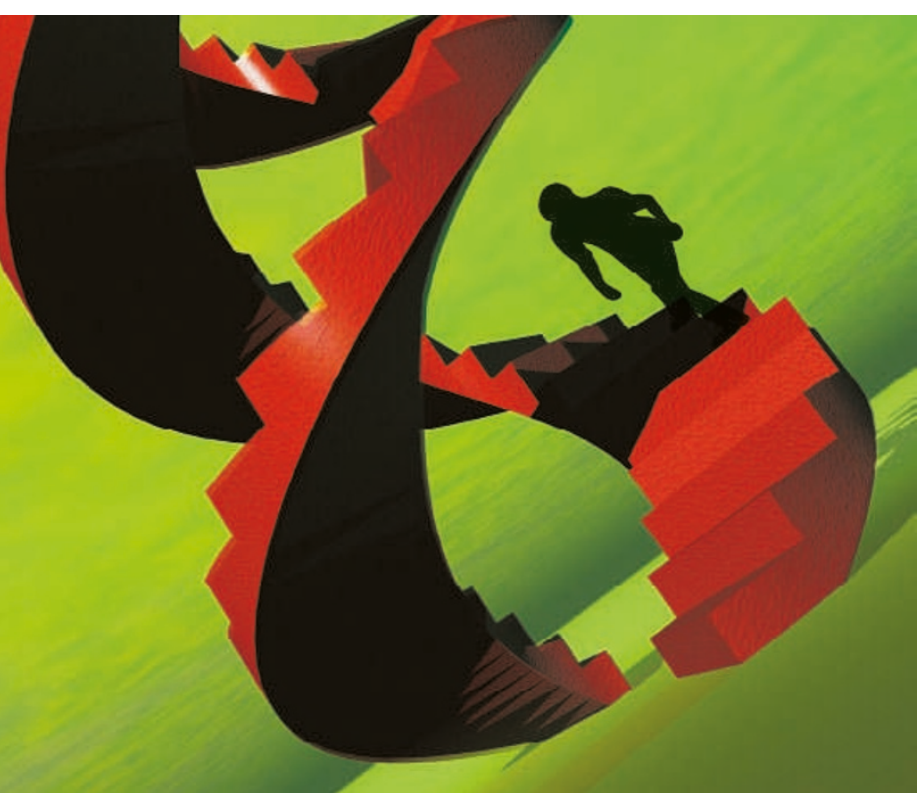

neurotrophic factor (BDNF) pathway has opposing effects on cocaine reward in the two main populations of neurons of the NAc.

The NAc contains medium spiny neurons (MSNs), which project to downstream structures and are divided into two populations on the basis of their expression of D1 or D2 dopamine receptors. These two populations have antagonistic outputs.

Cocaine exposure upregulates BDNF, and its receptor neurotrophic tyrosine kinase receptor type 2 (TrkB) in the NAc, so the authors investigated whether BDNF and TrkB have distinct roles in the two types of MSN. They used strains of mice in which the expression of TrkB could be selectively and conditionally turned off in MSNs containing either D1 or D2 receptors.

When TrkB was turned off in D1-containing MSNs, the mice showed an increased cocaine reward response in a conditioned place preference test and an increased locomotor sensitization to cocaine. Neuronal activity in the NAc shell
- as measured by FOS expression was decreased but the neuronal excitability remained unchanged. Lack of TrkB in D2-containing MSNs had the opposite effect and caused increased excitability.

Next, the authors introduced a blue-light-sensitive cation channel (ChR2) into D1- and/or D2-containing MSNs in the NAc, which allowed the neurons to be selectively activated by blue light shining through a cannula into the NAc. Light-induced activation of D1-containing MSNs or of both types of MSNs induced an increase in cocaine preference in the mice. By contrast, activation of D2-containing MSNs alone decreased preference for cocaine, mimicking the effects of TrkB deletion.

Together, the results are consistent with a model in which activation of D2-containing MSNs in the NAc antagonizes the cocaine reward response, whereas activation of D1-containing MSNs potentiates it. Changes in the balance between the two circuits might contribute to the addicted state, and these findings might therefore provide a target for treatment of addiction.

Rachel Jones

ORIGINAL RESEARCH PAPER Lobo, M. K. et al. Cell-type specific loss of BDNF signaling mimics optogenetic control of cocaine reward. Science 330, 385-390 (2010) 